Antje Wiener

\title{
Kontestation in verknüpften Diskursräumen
}

Der Aufsatz argumentiert für den Fortbestand der Zeitschrift für Internationale Beziehungen, um den erfolgreichen Zugang zur Gestaltungsmacht zentraler Themen und Fragestellungen im globalen Zusammenhang weiterhin zu gewährleisten. Das Argument basiert auf einem Doppelschritt: erstens wird mittels Debatten in der ZIB ein Thema in der deutschsprachigen IB-Gemeinde platziert. Dieses wird dann zweitens durch die Publikation im englischsprachigen Kontext in der globalen IB-Gemeinde präsentiert. Diese Stabilisierung ist jedoch nicht allein der quantitativen Verbreiterung der Debatte, sondern der qualitativen Vertiefung durch Zugang zur Gestaltungsmacht im Universum des IB-Diskurses durch die schrittweise Nutzung und Verknüpfung unterschiedlicher Sprachräume geschuldet. Grundlage für die Argumentation ist daher die Funktion der ZIB als Diskussionsraum, der Kontestationen innerhalb der ZIB und darüber hinaus fördert. Hier wird durch die Verknüpfung über nachgeordnete Schritte Zugriff auf die Entfaltung globaler Debatten ermöglicht. Der Aufsatz entwickelt dieses Argument mit Bezug auf drei zentrale Debatten der ZIB.

\section{Einleitung}

Aus der Perspektive des englischsprachigen Auslands während der ersten eineinhalb Dekaden hat sich die Zeitschrift für Internationale Beziehungen (ZIB) vor allem durch »freundschaftliche Debatten« mit gesellschaftstheoretischem Tiefgang und internationaler Relevanz hervorgetan. ${ }^{1}$ Damit wurde als Alternative zu den Pseudo-Debatten, bei denen die »Gesprächspartner« (sic!) vorzugsweise nicht aufeinander trafen (Lapid 1989; Waever 1996), ein neuer interaktiver Diskurs im Universum des internationalen IB-Diskurses eingeführt. ${ }^{2}$ So weckte die Diskussion aus Deutschland zunächst Neugier. Warum diese deutsche Debattenkultur im Universum des IB-Diskurses wichtig ist und daher einen festen Platz behalten sollte, wird im Folgenden unter Verwendung eines normgenerativen Kontestationsbegriffs (vgl. Wiener 2014: Kap. 1) erläutert.

Zusammengefasst argumentiert der Beitrag, dass die Sichtbarkeit der ZIB zunächst bei deutschsprachigen und schließlich auch anderen Wissenschaftlerinnen

1 Das Konzept der friendly debate wurde dann auch nicht überraschend von zwei deutschsprachigen IB-ler/innen im Ausland im Rahmen der beginnenden Debatte zwischen Rational Choice und Konstruktivismus erwähnt. Vgl. Risse und Wiener (1999: 775-782).

2 Zum Konzept des Diskursuniversum als Raum, in dem Macht durch Verteilungskämpfe umstritten ist, vergleiche Jane Jensons »universe of political discourse« (1989: 235-258), zur Anwendung des Konzepts in den IB vgl. Sandra Whitworths Beiträge zur Third Debate (1989: 265-272). 
und Wissenschaftlern im Ausland vor allem durch die Kombination einer ausgesprochen effektiven Praxis hergestellt werden konnte. Diese Entwicklung begründet sich in einem Doppelschritt: So wurde einerseits Sichtbarkeit durch Debatten innerhalb der ZIB beziehungsweise der deutschsprachigen IB-Gemeinde erzielt. Andererseits konnte die Stabilisierung durch Publikation der Debattenthemen in einer englischsprachigen Umgebung und der entsprechenden Verankerung im englischsprachigen ausländischen Umfeld hergestellt werden. Diese Stabilisierung ist jedoch - so mein zweiter Argumentationsgang - nicht allein der quantitativen Verbreiterung der Debatte beispielsweise durch die nahtlose Eingliederung der ZIB in die englischsprachige internationale IB-Gemeinschaft geschuldet. Sondern sie fußt in der qualitativen Vertiefung durch Zugang zur Gestaltungsmacht im Universum des IB-Diskurses durch die schrittweise Nutzung und Verknüpfung unterschiedlicher Sprachräume. Im Folgenden soll dieses Argument in zwei Schritten kurz skizziert werden: Der erste Abschnitt detailliert das Argument und fasst den Erfolg bisheriger Debatten im Lichte der Kontestationen zusammen; der zweite Abschnitt verweist auf die ersten Resultate und zielt auf eine Verstetigung des Zugangs zu normgenerativer Kontestation im Rahmen wissenschaftlicher Praxis als mittelfristiger Publikationsstrategie der ZIB ab.

\section{Zugang zur Gestaltung von Debatten}

Die Praxis der Kontestation in zwei Etappen hat bisher recht erfolgreich Diskursräume im In- und Ausland verknüpft. Ohne Grenzziehungen stützen zu wollen, sollte die Unterteilung in unterschiedliche Diskursräume jedoch durchaus als Vorteil begriffen und als solcher entsprechend strategisch ausgestaltet werden. Denn gerade durch die schrittweise Entfaltung von Debatten wird die Ausweitung des Zugangs zur Gestaltungsmacht auf verschiedene Gruppen innerhalb der IB und damit ein pluralistischer Blick auf die Theoriegestaltung gefördert. So zeigt sich bereits beim kursorischen Querlesen beispielsweise eine Öffnung des Zugangs hinsichtlich der Gender-Balance von einem vornehmlich männlich geprägten Zugang zum Diskursuniversum in der ersten ZIB-Debatte hin zu einem stärker durch Wissenschaftlerinnen gestalteten Aufschlag der gegenwärtigen dritten ZIB-Debatte (vgl. dazu u.a. Hofius et al., in diesem Heft). Die anschließende Rekonstruktion der Debattenschwerpunkte fasst die Zugangskriterien sowie ihre Veränderung über die letzten zwei ZIB-Dekaden im Folgenden kurz zusammen.

\subsection{Gestaltungsmacht im Universum des IB-Diskurses}

Während der ersten Etappe der Kontestation überwog der wissenschaftliche Austausch unter deutschsprachigen Kolleginnen. Denn nur diese hatten die notwendigen Kapazitäten, um auch den vollumfänglichen Zugang zum Diskurs zu praktizieren. Dieser ist jedoch zentral für die Akzeptanz und entsprechend auch für die Rolle 
der ZIB innerhalb internationaler Diskursgemeinschaften. Gleichzeitig bleibt die Gestaltungsmöglichkeit wissenschaftlicher Debatten mit Bezug auf und Relevanz für das globale Universum des IB-Diskurses durch die Teilnahme im deutschsprachigen Kontext maßgeblich eingeschränkt. Wie die Citizenship Theorie zeigt, ist ohne diesen Zugang jedoch nur ein zweitklassiger Mitgliedschaftsstatus ohne Gestaltungsmacht erreichbar. Diese Argumentation basiert auf dem Zugangskonzept nach Thomas H. Marshall und ist entsprechend in dem Verständnis von Bürgerschaft als historisch kontingente Praxis begründet. ${ }^{3}$ Entsprechend einer praxisorientierten Anwendung des Konzepts von (Staats-)Bürgerschaft auf die Teilnahme am IB-Diskurs bedeutet dies, dass nur diejenigen, die auch die deutsche Sprache beherrschen, sich vollumfänglich in die Debatte einbringen können. Das heißt, erst der Zugang durch Sprache erlaubt es, das Recht zur Partizipation auch auszuüben und entsprechend diskursive Gestaltungsmacht aufgrund von voller Mitgliedschaft in der Diskursgemeinschaft zu entfalten. Damit war die erste - deutschsprachige Etappe der normgenerativen Kontestation erfolgreich abgeschlossen.

Jedoch ermöglichte erst die zweite - englischsprachige - Etappe den Zugang auch für wissenschaftliche Beiträge innerhalb der internationalen IB-Gemeinschaft. Das heißt, erst der Zugang zu Kontestation sowohl innerhalb wie auch außerhalb der ZIB hat dazu beigetragen, die ZIB als Diskursraum im Rahmen der internationalen Publikationsumgebung nachhaltig zu stabilisieren. Angesichts dieser Beobachtung ist zu überlegen, wie diese effektive Praxis der über zwei Etappen beziehungsweise zwei Diskursräume verknüpften Kontestation mittelfristig als Publikationsstrategie der ZIB konstruktiv aufzunehmen und proaktiv zu entfalten wäre. Wenn es der ZIB entsprechend gelänge, die bisherige Praxis der Ausgestaltung und Verknüpfung von Diskussionsräumen strategisch umzusetzen, wäre eine globale Sichtbarkeit zu erlangen. Daher wäre es sinnvoll, die Rolle eines deutschsprachigen Publikationsorgans mittelfristig erfolgreich zu verstetigen. Die folgenden Abschnitte skizzieren dies mit Bezug auf die bisherige 20jährige Publikationspraxis.

\subsection{Kommunikatives Handeln: Rational oder konstruktivistisch?}

Die erste, bisher umfangreichste ZIB-Debatte bildete die Grundlage einer solchen etappenweisen Kontestation. Damit wurden neue Möglichkeiten deutschsprachiger Publikation in der aktiven Ausgestaltung des globalen IB-Diskurses durch die Verknüpfung von Diskussionsräumen deutlich. Die Bedeutung der Debatte der Habermasschen Theorie des kommunikativen Handelns und ihrer Anwendung in den internationalen Beziehungen ${ }^{4}$ ist für die Weiterentwicklung der international geführten »Neo-Neo«-Debatten um die Kooperationstheorien und die Regimeforschung

3 Vgl. dazu Marshalls Beispiel der Entwicklung von Bürgerschaftsrechte über zwei Jahrhunderte hinweg in Großbritannien als Triade ziviler, politischer und sozialer Rechte (1950).

4 Siehe hierzu die zahlreichen ZIB-Beiträge in den Jahren 1994 und 1995 (siehe insbesondere Müller, Risse-Kappen, Hellmann, Gehring und Zürn). 
der 1980er hinreichend bekannt. ${ }^{5}$ Darüber hinaus hat sie in den 1990er Jahren die Debatte um die Ausrichtung von Theorien mittlerer Reichweite, die vor allem zwischen Vertreterinnen von Rational Choice Ansätzen und konstruktivistischen Ansätzen in den IB und in der europäischen Integrationsforschung geführt wurden, reflektiert. Ihr Erfolg ist vor allem der zentralplatzierten englischsprachigen Publikation ihrer Inhalte geschuldet. ${ }^{6}$ Darüber hinaus hat die Frankfurter Konferenz zum Thema und die Publikation der Beiträge in dem Suhrkamp Band »Anarchie der kommunikativen Freiheit: Habermas und die internationale Politik« durch Peter Niesen und Benjamin Herborth (Herborth/Niesen 2007) ergebnisorientiert eine Grundlage für die Herausbildung einer Internationalen Politischen Theorie auch in Deutschland legen können. Dies wurde wiederum von der ZIB durchaus strategisch aufgegriffen. ${ }^{7}$ Zusammengefasst hat sich durch diese erste große ZIB-Debatte eine neue internationale Sichtbarkeit der deutschen IB etabliert, im Zuge derer die externe Wahrnehmung der deutschsprachigen IB sich radikal veränderte: Statt vereinzelter kritischer Beiträge - beispielsweise von Krippendorf (1987: 235-258) oder Czempiel (Rosenau/Czempiel 1992) ${ }^{8}$ - wurden jetzt zunehmend die Publikationen deutscher Wissenschaftlerinnen und Wissenschaftler als Kerntexte international geführter Debatten zitiert.

\subsection{Integrationstheorie: Postmodern oder nicht?}

Bei der folgenden Debatte geht es um die Diskussion über die neue Faszination mit postmodernen Ansätzen in den IB sowie in der europäischen Integrationsforschung, die von Thomas Diez (1996) begonnen und von Tanja Börzel (1997: 125-137) aufgenommen wurde und anschließend vor allem von Diez durch seine zahlreichen Beiträge zum Thema international verankert wurde (vgl. vor allem Diez 1998; Diez et al. 2008; Diez 2013: 194-210). Wenn sie auch weniger intensiv in Deutschland entfaltet wurde, so konnte doch durch die Prominenz und Präsenz der beteiligten Autor/innen und ihrer Publikationen in englischsprachigen Zeitschriften ebenfalls zumal aus der Auslandsperspektive - durchaus die Sichtbarkeit der ZIB gestärkt werden. Damit war auch diese Debatte prägend für die Rolle der Zeitschrift als ein Ort im Universum des IB-Diskurses an dem unterschiedliche Positionen diskutiert werden.

5 Siehe dazu vor allem die teils beißende Kritik in der Beobachtung durch Ole Waever, der in der Debatte zwischen Neoliberalen und Neorealisten der englischsprachigen IB die »boundary of boredom« überschritten sah (Waever 1996: 23).

6 Vgl. dazu vor allem die erste Zusammenfassung durch Thomas Risse (2000: 1-39) sowie die ebenfalls besonders sichtbaren Publikationen von u.a. Harald Müller (2004: 395-435), Nicole Deitelhoff und Harald Müller (2005: 167-179).

7 Vergleiche dazu die Symposiumsbeiträge von Brunkhorst, Deitelhoff und Wiener in der ZIB 2010.

8 Dies waren in den 1990er Jahren in der Tat die einzigen deutschen Beiträge, die in den $\mathrm{PhD}$ Kursen beispielsweise an kanadischen Universitäten wie der Carleton University auf der Reading List auftauchten. 


\section{Neue Leuchttürme im IB-Diskurs}

\subsection{Kritische Normenforschung: Detailliert oder Reflektiert?}

Ob die sich derzeit entfaltende Debatte um die Ausgestaltung kritischer Normenforschung zur »Normativität von Normen $\aleph^{9}$ eine ähnlich breite internationale Wirkung erzielt, steht noch offen. Wünschenswert wäre diese Entwicklung. Denn die Ausgestaltung der Forschung zu dieser Forschungslücke der internationalen Normenforschung bietet beträchtliches Potenzial sowohl für die Entfaltung der Debatte im deutschen Kontext als auch für die Verankerung im englischsprachigen ausländischen Publikationskontext. Zwar wurde die von Stephan Engelkamp, Katharina Glaab und Judith Renner (2012) angestoßene Debatte um die Bodenhaftung kritischer Normenforschung innerhalb der ZIB von deutschsprachigen Autor/innen aufgenommen. Anders als die erste große Debatte um die Theorie des kommunikativen Handelns wird sie jedoch nicht maßgeblich mit Referenz auf deutsche Gesellschaftstheoretiker (sic!) geführt. Stattdessen werden international publizierte englischsprachige Referenzen zu laufenden Debatten in der Normenforschung herangezogen, die sich mit der Frage der Normativität von Normen und dem Zusammenwirken von normativer Theorie und der empirischen Untersuchung von Normwirkung beschäftigen. ${ }^{10}$ Letztendlich wird diese Debatte von einer dezidierten Auseinandersetzung mit dem Konzept der Bifokalität, das die Wechselwirkung von normativer und empirischer Forschungsperspektive zum Ausgangspunkt kritischer Normenforschung macht, profitieren. ${ }^{11}$ Während die reflexive kritische Normenforschung es sich zur Aufgabe macht, durch diese bifokale methodologische Ausrichtung die Entstehung und Wirkung der Normativität von Normen durch empirische Forschung zu substantiieren, läuft das von Engelkamp et al. sogenannte »Programm« zur kritischen Normenforschung allerdings eher Gefahr, durch die überwiegend empirische Schwerpunktsetzung, die Themen der Kontingenz und Begründung von Normativität zu umschiffen.

Diese sind jedoch vor allem in Hinblick auf die empirisch noch kaum systematisch erforschte Wechselwirkung der Umsetzung normativer Bedeutungsstrukturen und ihrer internationalen Folgen für die großen Fragen der »Governance in Räumen begrenzter Staatlichkeit« (Risse/Lehmkuhl 2007: 144-159) sowie der »globalen Konstitutionalisierung« (Dunoff/Trachtman 2009) von besonderer Relevanz. Hier

9 Vgl. dazu derzeit den Beitrag von Engelkamp, Glaab und Renner (2012: 101-128), die Reaktion von Deitelhoff und Zimmermann (2013), die Replik von Engelkamp, Glaab und Renner (2013) sowie den jüngsten Beitrag von Hofius et al., in diesem Heft.

10 Vgl. die Verweise auf Richard Price (2008: 317-326). Leider bleiben Engelkamp et al. jedoch hinter dem Stand der Literatur, die im Rahmen der - übrigens sicher nicht erst 2013 begründeten - kritischen Normenforschung beispielsweise an die frühen Arbeiten von Kratochwil (1984) anschließt, weit zurück. Für eine umfassendere Referenz zur Literatur vgl. Hofius, Gholiagha und Hansen-Magnusson (2014).

11 Vgl. zur Anwendung der bifokalen Forschungsperspektive aus der Public Philosophy in der kritischen Normenforschung Wiener (2008), Deitelhoff (2009: 33-65), Deitelhoff und Zimmermann (2013). 
sollte entsprechend die Chance zur Ausgestaltung neuer Leuchttürme nicht versäumt werden, denn sie eröffnet unter anderem für die ZIB ein diskursstrategisches Gelegenheitsfenster: Erstens sind gut aufgestellte internationale Netzwerke in der kritischen Normenforschung vorhanden; zweitens sind in den IB bisher zu keinem Zeitpunkt so hervorragend methodologisch ausgebildete jüngere Kolleginnen und Kollegen am Start wie jetzt (die neueste ZIB-Debatte sowie auf den Markt strebende Dissertationen belegen das nachdrücklich); und drittens war die politische Theorie selten so bereit zur normativen Interaktion mit der IB wie derzeit. Dies ist zwar nicht nur, aber doch maßgeblich der international sichtbaren Innovation einer nachrückenden Generation (vor allem in Frankfurt/Main, Münster und Hamburg als zentralen Austragungsorten der vom Netzwerk kritischer Normenforschung organisierten Tagungen) zu verdanken.

\subsection{Perspektiven}

Angesichts dieser positiven Erfahrungen wäre die ZIB gut beraten, die Praxis der Verknüpfung von Diskussionsräumen durch Etappen der Kontestation in unterschiedlichen Sprachkontexten beizubehalten. Wenn es weiterhin gelingt, die Diskussionsräume zu wahren, in denen in deutscher Sprache international relevante Themen ausformuliert werden und somit der Zugang zur detaillierten Auseinandersetzung mit diesen Themen nicht nur für die deutschsprachige IB-Gemeinschaft ermöglicht wird, bedeutet dies zudem eine Anreicherung spezifischer Kenntnisse in der deutschsprachigen IB. Damit erweitern sich nicht nur die Handlungsoptionen deutscher Wissenschaftlerinnen und Wissenschaftler. Denn durch die strategische Förderung dieser spezifischen Debattenkultur erhält die ZIB die Möglichkeit, sich in der zunehmend wettbewerbsorientierten globalen IB sichtbar zu präsentieren sowie darüber hinaus, das Universum des internationalen IB-Diskurses nachhaltig zu strukturieren und auszugestalten. ${ }^{12}$

\section{Literatur}

Börzel, Tanja 1997: Zur (Ir-)Relevanz der Postmoderne für die Integrationsforschung. Eine Replik auf Thomas Diez Beitrag »Postmoderne und europäische Integration«, in: Zeitschrift für Internationale Beziehungen 4: 1, 125-137.

Brunkhorst, Hauke 2010: Neustart - Kritische Theorie Internationaler Beziehungen, Zeitschrift für Internationale Beziehungen 17: 2, 293-315.

Deitelhoff, Nicole/Müller, Harald 2005: Theoretical Paradise - Empirically Lost? Arguing with Habermas, in: Review of International Studies 31: 1, 167-179.

12 Dies wird unter anderem durch die doppelte Publikation von ZIB-Debattenbeiträgen in globalen Publikationsmedien gefördert (vgl. die Wiederveröffentlichung der englischsprachigen Übersetzung der Beiträge zur derzeitigen ZIB-Debatte in englischer Sprache im World Political Science Review 2014). 
Deitelhoff, Nicole 2009: The Discursive Process of Legalization: Charting Islands of Persuasion in the ICC Case, in: International Organization 63: 1, 33-65.

Deitelhoff, Nicole 2010: Parallele Universen oder Verschmelzung der Horizonte?, in: Zeitschrift für Internationale Beziehungen, 17: 2, 279-292.

Deitelhoff, Nicole/Zimmermann, Lisbeth 2013: »Things We Lost in the Fire: How Different Types of Contestation Affect the Validity of International Norms« (HSFK Arbeitspapiere 18), Frankfurt/Main.

Diez, Thomas 1996: Postmoderne und europäische Integration. Die Dominanz des Staatsmodells, die Verantwortung gegenüber dem Anderen und die Konstruktion eines alternativen Horizonts, in: Zeitschrift für Internationale Beziehungen 3: 2, 255-81.

Diez, Thomas 1998: Perspektivenwechsel: Warum ein »postmoderner « Ansatz für die Integrationsforschung doch relevant ist, in: Zeitschrift für Internationale Beziehungen 5: 1, 139-148

Diez, Thomas/Albert, Mathias/Stetter, Stephan (Hrsg.) 2008: The European Union and Border Conflicts. The Power of Integration and Association, Cambridge.

Diez, Thomas 2013: Normative Power as Hegemony, in: Cooperation and Conflict 48: 2, 194-210.

Dunoff, Jeffrey L./Trachtman, Joel P. (Hrsg.) 2009: Ruling the World? Constitutionalism, International Law, and Global Governance, Cambridge.

Engelkamp, Stephan/Glaab, Katharina/Renner, Judith 2012: In der Sprechstunde: Wie (kritische) Normenforschung ihre Stimme wiederfinden kann, in: Zeitschrift für Internationale Beziehungen 19: 2, 101-128.

Engelkamp, Stephan/Glaab Katharina/Renner, Judith 2013: Ein Schritt vor, zwei Schritte zurück? Eine Replik auf Nicole Deitelhoff und Lisbeth Zimmermann, in: Zeitschrift für Internationale Beziehungen 20: 2, 103-116.

Gehring, Thomas 1994: Der Beitrag von Institutionen zur Förderung der internationalen Zusammenarbeit. Lehren aus der institutionellen Struktur der Europäischen Gemeinschaft, in: Zeitschrift für Internationale Beziehungen, 1: 2, 211-242.

Herborth, Benjamin/Niesen, Peter (Hrsg.) 2007: Anarchie der kommunikativen Freiheit. Jürgen Habermas und die Theorie der internationalen Politik, Frankfurt am Main.

Hellmann, Gunther 1994: Für eine problemorientierte Grundlagenforschung: Kritik und Perspektiven der Disziplin »Internationale Beziehungen« in Deutschland, in: Zeitschrift für Internationale Beziehungen 1: 1, 65-90.

Hofius, Maren/Gholiagha, Sassan/Hansen-Magnusson, Hannes 2014: Meaning-in-Use: Zum Verhältnis von Normativität und Normalität in der Normenforschung, in: Engelkamp, Stephan/Graf, Antonia/Glaab Katharina (Hrsg.): Kritische Normenforschung in Deutschland. Neue Wege in den Internationalen Beziehungen, Baden-Baden, i.E.

Jenson, Jane 1989: Paradigms and Political Discourse: Protective Legislation in France and the United States Before 1914, in: Canadian Journal of Political Science/Revue Canadienne de Science Politique 22: 2, 235-258.

Kratochwil, Friedrich 1984: The Force of Prescription, in: International Organization 38: 4, 658-708.

Krippendorf, Ekkehart 1987: The Dominance of American Approaches in International Relations, in: Journal of International Studies 16: 2, 207-214.

Lapid, Yosef 1989: The Third Debate: On the Prospects of International Theory in a Post-Positivist Era, in: International Studies Quarterly 33: 3, 235-254.

Marshall, Thomas Humphrey 1950: Citizenship and Social Class and other Essays, Cambridge.

Müller, Harald 1994: Internationale Beziehungen als kommunikatives Handeln. Zur Kritik der utilitaristischen Handlungstheorien, in: Zeitschrift für Internationale Beziehungen 1: $1,15-44$.

Müller, Harald 2004: Arguing, Bargaining and All That: Communicative Action, Rationalist Theory and the Logic of Appropriateness in International Relations, in: European Journal of International Relations 10: 3, 395-435. 
Price, Richard 2008: »The Ethics of Constructivism«, in: Reus-Smit, Christian/Snidal, Duncan (Hrsg.): The Oxford Handbook of International Relations, Oxford, 317-326.

Risse, Thomas 2000: »Let's Argue!« Communicative Action in World Politics, in: International Organization 54: 1, 1-39.

Risse-Kappen, Thomas 1995: Reden ist nicht billig. Zur Debatte um Kommunikation und Rationalität, in: Zeitschrift für Internationale Beziehungen 2: 1, 171-184.

Risse, Thomas/Wiener, Antje 1999: »Something Rotten« and the Social Construction of Social Constructivism: A Comment on Comments, in: Journal of European Public Policy 6: 5, 775-782.

Risse, Thomas/Lehmkuhl, Ursula 2007: Governance in Räumen begrenzter Staatlichkeit: Anmerkungen zu konzeptionellen Problemen der gegenwärtigen Governance-Diskussion, in: Beisheim, Marianne/Schuppert, Gunnar Folke (Hrsg.): Staatszerfall und Governance, Baden-Baden, 144-159.

Rosenau, James N./Czempiel, Ernst-Otto 1992: Governance without Government: Order and Change in World Politics, Cambridge.

Waever, Ole 1996: The Rise and Fall of the Inter-Paradigm Debate, in: Smith, Steve/Booth, Ken/Zalewski, Marysia (Hrsg.): International Theory: Positivism and Beyond, Cambridge.

Whitworth, Sandra 1989: Gender and the Inter-Paradigm Debate. Millennium 18: 2, 265-272.

Wiener, Antje 2008: The Invisible Constitution of Politics: Contested Norms and International Encounters, Cambridge.

Wiener, Antje 2010: Zur normativen Wende in den IB: Triangulation of a Different Kind, in: Zeitschrift für Internationale Beziehungen 17: 2, 335-354.

Wiener, Antje 2014: A Theory of Contestation, Heidelberg.

Zürn, Michael 1994: We Can Do Much Better! Aber muß es auf amerikanisch sein? Zum Vergleich der Disziplin »Internationale Beziehungen« in den USA und in Deutschland, in: Zeitschrift für Internationale Beziehungen 1: 1, 91-114. 